\title{
NEW BEAM DIAGNOSTIC DEVELOPMENTS AT THE PHOTO-INJECTOR TEST FACILITY PITZ*
}

\author{
S. Khodyachykh ${ }^{\dagger}$, G. Asova, J. W. Bähr, C. Boulware, H.-J. Grabosch, M. Hänel, S. Korepanov, \\ S. Lederer, M. Krasilnikov, A. Oppelt ${ }^{\ddagger}$, B. Petrosyan, S. Rimjaem, \\ T. Scholz, R. Spesyvtsev, L. Staykov, F. Stephan (DESY, Zeuthen, Germany), \\ T. Garvey (LAL, Orsay, France), J. Rönsch (Universität HH, Hamburg, Germany), \\ D.J. Holder, B. Muratori (STFC/DL/ASTeC, Daresbury, Warrington, Cheshire, UK), \\ L. Hakobyan (YerPhI, Yerevan, Armenyia), D. Alesini, L. Ficcadenti (INFN/LNF, Frascati, Italy), \\ D. Richter (BESSY, Berlin, Germany)
}

\begin{abstract}
The Photo-Injector Test Facility at DESY in Zeuthen (PITZ) is an electron accelerator which was built to develop and optimize high brightness electron sources suitable for SASE FEL operation. Currently, in parallel to the operation of the existing setup, a large extension of the facility and its research program is ongoing. The beam line which has a present length of about 13 meters will be extended up to about 21 meters within the next year. Many additional diagnostics components will be added to the present layout. Two high-energy dispersive arms, an RF deflecting cavity and a phase space tomography module will extend the existing diagnostic system of the photo injector and will contribute to the full characterization of new electron sources. We report on the latest developments of the beam diagnostics at PITZ.
\end{abstract}

\section{INTRODUCTION}

The test facility PITZ [1] was built in Zeuthen in collaboration with international partners with the goal to develop and to optimize high brightness electron sources suitable for SASE FEL operation, to compare detailed experimental results with numerical simulations as well as to test new developments such as laser system, photo cathodes and new beam diagnostic elements.

\section{PRESENT BEAM DIAGNOSTICS AT PITZ}

The PITZ facility is an electron accelerator which consists of a 1.5 cell L-band RF gun with its photo-cathode laser system, followed by a low energy diagnostics section, a normal-conducting booster cavity and a high energy diagnostics section. Presently both, low and high energy diagnostics sections serve for the precise measurements of the following electron beam parameters:

Beam size, shape and position using YAG or OTR view screen stations combined with the CCD Cameras or

* This work has partly been supported by the European Community, contracts RII3-CT-2004-506008 and 011935, and by the 'Impuls- und Vernetzungsfonds' of the Helmholtz Association, contract VH-FZ-005.

† sergiy.khodyachykh@ desy.de

$\ddagger$ Presently at Paul Scherrer Institut, 5232 Villigen PSI, Switzerland.

06 Instrumentation, Controls, Feedback \& Operational Aspects using one of two installed wire scanners;

Beam charge using Faraday cups or integrating current transformators (ICT);

Bunch length using aerogel or OTR as radiators, and optical transmission line and a streak camera;

Transverse emittance using three emittance measuring systems (EMSYs) employing the slit scan method;

Beam momentum and momentum spread using a dipole magnet and a view screen at low energy (after the gun) or at high energy (after the booster);

Longitudinal phase space for this type of measurements low energy dispersive arm is equipped with an aerogel radiator and a readout for streak camera.

\section{FUTURE BEAM DIAGNOSTICS AT PITZ}

In parallel to beam operation we are permanently extending the diagnostic section in order to enable more detailed studies of the beam properties. The beam line which has a present length of about 13 meters will be extended up to about 21 meters towards the middle of 2008. A schematic layout of the future PITZ beam line is shown in Fig. 1. Several additional diagnostic components will be added to the present setup. Together with the new first high-energy dispersive arm (HEDA1), the deflecting cavity (RFD), and the phase space tomography module (PST), the second highenergy dispersive arm HEDA2 will extend the existing diagnostics system of the PITZ facility. At the beginning of 2008 a new normal conducting booster cavity will run into operation. This will reflect in an increase of the maximum beam momentum. Thus, all new diagnostics elements are designed to operate at beam momenta up to $40 \mathrm{MeV} / \mathrm{c}$.

\section{Upgrade of LEDA}

The low energy dispersive arm (LEDA) is a $60^{\circ}$ bending magnet located about $1 \mathrm{~m}$ downstream the cathode. This magnet is a copy of the dipole magnet used at FLASH facility. Contrary to FLASH, at PITZ only one booster cavity is used for the further acceleration of the beam. For solenoid current settings which correspond to the focusing of the beam downstream of the booster cavity, the beam

T03 Beam Diagnostics and Instrumentation 

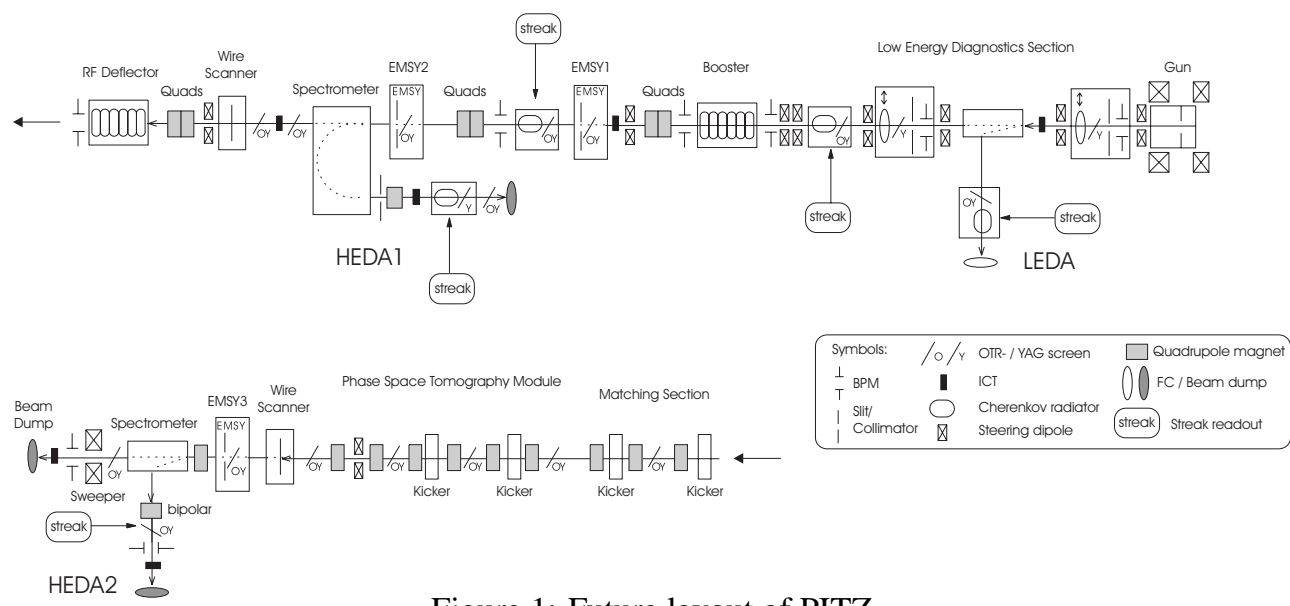

Figure 1: Future layout of PITZ.

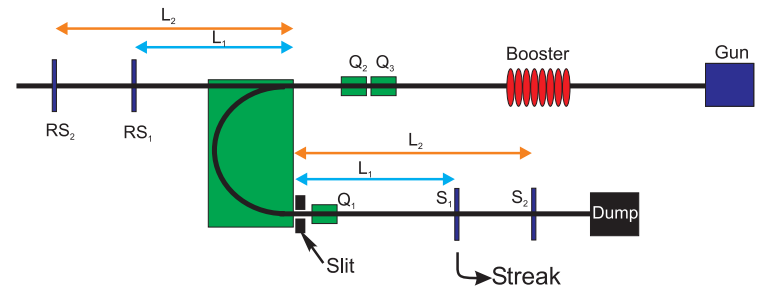

Figure 2: Simplified layout of PITZ with the first high energy dispersive section.

size at the position of the dipole magnet is larger than the vacuum chamber. Thus, the main drawback of the present magnet is the small inner size of the vacuum chamber of only $12 \mathrm{~mm}$ caused by a small distance between the pole shoes of only $20 \mathrm{~mm}$. In the nearest future it is planned to re-machine the pole shoes of the dipole magnet of the LEDA and to re-design its vacuum chamber in order to increase the aperture of the vacuum chamber.

Moreover, in order to improve the resolution of the momentum measurements of the low energetic beam we are planning to use a slit upstream the mentioned dipole magnet. For this purpose the new actuator with the slit is under construction for the so-called double-diagnostic-cross (DDC). Moreover, the upgraded geometry of the DDC will improve the vacuum conditions and the situation with wake fields. The new DDC will be installed during the next shutdown.

\section{HEDAI}

The first high energy dispersive arm HEDA1 will be placed after the booster cavity, at about $7 \mathrm{~m}$ downstream the gun. This multipurpose device is designed [2] and will be put into operation in autumn 2007. It combines the functionality of (i) an electron spectrometer, (ii) a device for the characterization of the longitudinal phase space, and (iii) a transverse slice emittance measuring system. The layout of HEDA1 is schematically shown in Fig 2. It consists of a 180 degree dipole magnet having the bending radius of $300 \mathrm{~mm}$ followed by a slit, a quadrupole magnet, and two screen stations. One of the screen stations will be equipped with an optical read-out for a streak camera for investiga06 Instrumentation, Controls, Feedback \& Operational Aspects tions of the longitudinal phase space [3]. The functionality of HEDA1 will be enhanced with a setup that allows to measure the transverse emittance of the electron beam at different longitudinal positions along the bunch. The socalled slice emittance provides better understanding of the physics of a photoinjector, particulary the emittance compensation and conservation principles. Using proper phasing of the booster cavity, one can obtain linear correlation between the momentum and longitudinal distribution of the electrons in the bunch. A slit at the dipole exit selects the necessary slice from the energy chirped beam. This slice is scanned with the quadrupole magnet focusing in a plane orthogonal to the dispersion plane and the beam distribution is observed on screen.

The main advantage of the spectrometer based on a $180^{\circ}$ dipole magnet is the simplicity to reconstruct the momentum distribution [4]. One uses the reference screen $R S_{i}$ in the straight section and measures the contribution from the transverse beam size and divergence, which can be deconvoluted with the spectrum measured on the corresponding screen $S_{i}$ to obtain the pure momentum distribution. This deconvolution is simple and straightforward if the distances $L_{1}\left(L_{2}\right)$ from the entrance of the dipole magnet to the measuring $S_{1}\left(S_{2}\right)$ and the reference $R S_{1}\left(R S_{2}\right)$ screens are equal. For this reason reference screen stations $R S_{1}$ and $R S_{2}$ are placed downstream of the dipole magnet in the main beam line. Additional components which contribute to the measurements at HEDA1 are two quadrupole magnets $Q_{2}$ and $Q_{3}$ located in front of the dipole magnet entrance. By focusing the beam on the screen $R S_{i}$ with the help of these quadrupole magnets one controls the resolution of the momentum measurement on the corresponding screen $S_{i}$. The quadrupole magnets $Q_{2}$ and $Q_{3}$ will also be used as a part of the matching section for the phase space tomography diagnostics described below. HEDA1 will be installed at PITZ during the shutdown of summer 2007.

\section{RF Deflecting Cavity}

For a detailed phase space analysis it is planned to install an RF deflecting cavity. Travelling through this cavity, the electron beam will get a time dependent vertical 
deflection, so that a strong correlation between longitudinal z-coordinate and transverse position is introduced. Further analysis of transverse and longitudinal phase space of the deflected beam can be done in the tomography section and in the second High Energy Dispersive Arm HEDA2 (both described below), respectively. Figure 3 shows a schematic layout of the RF deflector (RFD) followed by a tomography module. For the RF deflector a travelling

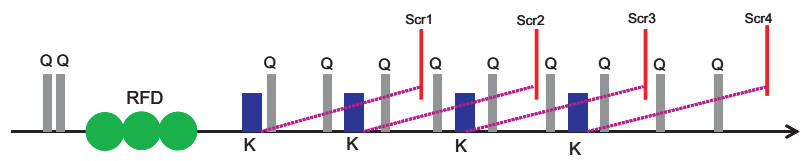

Figure 3: Simplified layout of the RF deflector followed by tomography section.

wave structure was chosen. It has small field filling time $(\sim 0.1 \mu \mathrm{s})$. This allows to analyze single bunches without significant distortion of the others in a train of up to 3250 bunches, as foreseen for the European XFEL. To distinguish between deflected and non-distorted bunches several kickers $\mathrm{K}$ which kick the deflected bunch in horizontal direction are foreseen downstream the deflecting cavity. The physical design of the deflecting cavity has been done. The RF deflector has to provide a deflecting voltage of up to 1.8 MV and should cause minimal distortion of the beam phase space in the measurement directions. We have compared two travelling wave structures operating at $1.3 \mathrm{GHz}$ and 3.0 GHz frequencies [5], showing a small advantage of the low frequency structure.

\section{Phase Space Tomography Section}

For detailed analysis of the transverse phase-space density distribution of the electron beam a phase space tomography section will be installed. The tomography section (Fig. 3) will consist of three FODO cells and four diagnostic stations for measuring the spatial beam density distributions. Several groups of quadrupole magnets distributed upstream along the PITZ beam line will be used to match the beam to the tomography section. Correct matching together with the proper geometry of the FODO cells and magnetic field strengths of its quadrupole magnets are chosen to deliver a phase advance of $45^{\circ}$ between the screens. Using modern tomographic algorithms one reconstructs the phase space distribution of the electron beam. Details of the physical design of the tomography section are discussed in [6]. The technical design is almost finalized and hardware components are partly ordered, so that the module will be installed at PITZ in early 2008.

\section{HEDA2}

The second high-energy dispersive arm (HEDA2) is in the process of physical design. Similarly to HEDA1, it will be used to measure the momentum distribution, the longitudinal phase space using a Cherenkov radiator and a streak camera and slice emittance. In addition the longitudinal phase space will be studied using the RF-deflector.
A major design request is to allow the measurement of the above mentioned beam parameters for bunch trains with up to 3250 pulses and a repetition rate of $10 \mathrm{~Hz}$ without significant temporal limitations of running hours. This requires a huge beam dump after the dispersive section, but due to the space restrictions the beam should be transported to the dump of the main beamline. The design considerations for this dispersive arm are discussed in details in [7].

\section{Streak Measurements}

A streak station for bunch length measurements after the booster was put into operation at the end of May 2007. The signal from the screen station (Cherenkov or OTR light) is transported to the streak camera by a $30 \mathrm{~m}$ long optical transmission line [3] and can be used for bunch length and longitudinal phase space measurements. In the future, using two quadrupole magnets placed directly after the booster, one will be able to conduct streak camera enhanced quadrupole scan for the slice emittance measurements. Future replacement of the refractive optics by reflective one will improve the temporal resolution of the system.

\section{CONCLUSION}

We described the new beam diagnostic developments at PITZ. Installation of the new components will open new possibilities in the characterization of the electron beam such as transverse slice emittance, phase space tomography and will provide an improved accuracy of the existing measurements, e.g. longitudinal phase space using an RF deflecting cavity.

\section{REFERENCES}

[1] F. Stephan et al., Photoinjector Test Facility under Construction at DESY Zeuthen, Proceedings of FEL 2000, Durham, NC, USA, 2000.

[2] S. Khodyachykh et al., Design and Construction of Multipurpose Dispersive Section at PITZ, Proceedings of DIPAC 2007, Venice, Italy, 2007.

[3] J.Bähr, J.Rönsch, Optical system for measurement of electron bunch length and longitudinal phase space at PITZ: extension and methodical investigations, Proceedings of DIPAC 07, Venice, Italy, 2007.

[4] S. Khodyachykh et al., Design of Multipurpose Dispersive Section at PITZ, Proceedings of FEL 2006, Berlin, Germany, 2006.

[5] S. Korepanov et al., An RF Deflector for the Longitudinal and Transverse Beam Phase Space Analysis at PITZ, Proceedings of DIPAC 2007, Venice, Italy, 2007.

[6] G. Asova et al., Design Considerations for Phase Space Tomography Diagnostics at the PITZ Facility, Proceedings of DIPAC 07, Venice, Italy, 2007, and references therein.

[7] J. Rönsch et al., Design Considerations of a Spectrometer Dipole Magnet for the Photo Injector Test Facility PITZ, Proceedings of DIPAC 07, Venice, Italy, 2007. 